\title{
A Rare Case of Ecchordosis Physaliphora Presenting With Headache, Abducens Nerve Palsy, and Intracranial Hypertension
}

\author{
Ruiqing Sun ${ }^{1}$, Yousaf Ajam ${ }^{1}$, Gerald Campbell ${ }^{2}$, Todd Masel ${ }^{1}$ \\ 1. Neurology, University of Texas Medical Branch, Galveston, USA 2. Pathology, University of Texas Medical Branch, \\ Galveston, USA
}

Corresponding author: Ruiqing Sun, rusun@utmb.edu

\begin{abstract}
We report a rare case of ecchordosis physaliphora presenting with headache, nausea, and diplopia. On neurological examination, the patient was found to have left abducens nerve palsy. CT of the head without contrast was unremarkable. Brain MRI demonstrated a non-enhancing retroclival mass with a mass effect upon the ventral pons. The mass had increased signal intensity on $\mathrm{T} 2$ and decreased signal intensity on T1weighted sequences. Lumbar puncture revealed an opening pressure of $37 \mathrm{~cm} \mathrm{H}_{2} \mathrm{O}$. The patient underwent an endoscopic endonasal approach for retroclival mass resection three weeks later. The tissue analysis of the mass was consistent with ecchordosis physaliphora. This could have been misdiagnosed as idiopathic intracranial hypertension had the MRI of the brain not been performed.
\end{abstract}

Categories: Neurology, Radiology, Neurosurgery

Keywords: ecchordosis physaliphora, headache, abducen nerve palsy, intracranial hypertension

\section{Introduction}

Ecchordosis physaliphora is a congenital benign hamartomatous lesion derived from notochord remnants. It is usually located in the retroclival prepontine region but can be found anywhere from the skull base to the sacrum [1]. Unlike chordomas, which are often symptomatic due to brainstem or cranial nerve compression, patients with ecchordosis physaliphora are usually asymptomatic. They are found in $0.5-2 \%$ of autopsies [2]. However, there are several case reports describing symptomatic ecchordosis physaliphora as well. The most common symptoms include headache, pneumocephalus, spontaneous cerebrospinal fluid (CSF) rhinorrhea with meningitis, and abducens nerve palsy [3-7]. The differential diagnoses of retroclival intradural lesions consist mainly of chordoma, ecchordosis physaliphora, skull base metastasis, dermoid cyst, epidermoid cyst, and arachnoid cyst. Distinguishing ecchordosis physaliphora from its malignant counterpart, chordoma, is very important because of the aggressive nature of the chordoma. CT is generally not sensitive for such lesions, primarily due to posterior fossa artifacts and the near-CSF density of the mass. The current goldstandard test for the diagnosis of ecchordosis physaliphora is brain MRI with and without contrast as well as pathology testing $[1,8,9]$. It is worth mentioning, however, that ecchordosis physaliphora and chordoma are

Received 05/22/2020

Review began 06/16/2020 Review ended 06/20/2020 Published 06/26/2020

\section{(c) Copyright 2020}

Sun et al. This is an open access article distributed under the terms of the Creative Commons Attribution License CC-BY 4.0., which permits unrestricted use, distribution, and reproduction in any medium, provided the original author and source are credited. histologically indistinguishable in small fragmentary specimens, and are generally differentiated by examining the margins, with the latter demonstrating infiltrative growth [1].

\section{Case Presentation}

Our patient was a 22-year-old female with a past medical history of headache who was admitted for severe headache, nausea, and diplopia. One day prior to presentation, the patient had awoken with a severe headache. She described her headache as located in the occipital region as well as the left frontal region and around the left eye. The associated symptoms were nausea, vomiting, and blurred vision, and there was no photophobia or phonophobia. The headache was aggravated by head movement, lying down, coughing, and bending over, and it was slightly alleviated by ibuprofen. The next day, when she awoke in the morning, she had blurred vision and binocular horizontal diplopia. Of note, the patient had been experiencing intermittent occipital headaches for almost one year, slightly relieved by over-the-counter oral analgesics [acetaminophen, nonsteroidal anti-inflammatory drugs (NSAIDS)]. She also reported a 30-lbs unintentional weight-gain over the past year. Neurological exam revealed impaired left eye abduction without papilledema or other neurological deficits. CT head without contrast was unremarkable (Figure $1 A, 1 B$ ). In order to rule out subarachnoid hemorrhage, a lumbar puncture was performed, which showed an opening

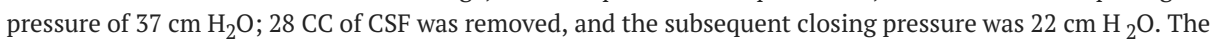
CSF examination was unremarkable (Table 1). Finally, brain MRI with and without contrast was performed and showed a non-enhancing retroclival mass with mass effect upon the ventral pons, which had increased signal intensity on $\mathrm{T} 2$ and decreased signal intensity on T1-weighted sequences, and was highly suspicious for ecchordosis physaliphora (Figure $2 A, 2 B, 2 C, 2 D$ ). Although the patient's headache had improved after the lumbar puncture, the diplopia persisted. The patient developed a post-lumbar puncture headache on the second day, and this resolved after intravenous hydration. She was discharged with a prescription for acetazolamide $500 \mathrm{mg}$ twice a day. 


\section{Cureus}

Three weeks later, the patient underwent an endoscopic endonasal approach for retroclival mass resection. The hematoxylin and eosin-stained, formalin-fixed, paraffin-embedded sections of the lesion showed physaliphorous cells and lymphocytic infiltrates in adjacent tissue; immunohistochemical stains showed strong reactivity of tumor cells for cytokeratin (AE1/AE3) and weak reactivity for S100. The surgical finding and tissue analysis of the mass were consistent with ecchordosis physaliphora (Figure 3). After surgery, at the two-month follow-up, her headache had resolved, but she had persistent diplopia; at five months, diplopia was found resolved and the patient was taken off Diamox (Teva

Pharmaceuticals, Parsippany, NJ); at eight months, the patient had no symptoms and a repeated brain MRI showed no tumor recurrence.

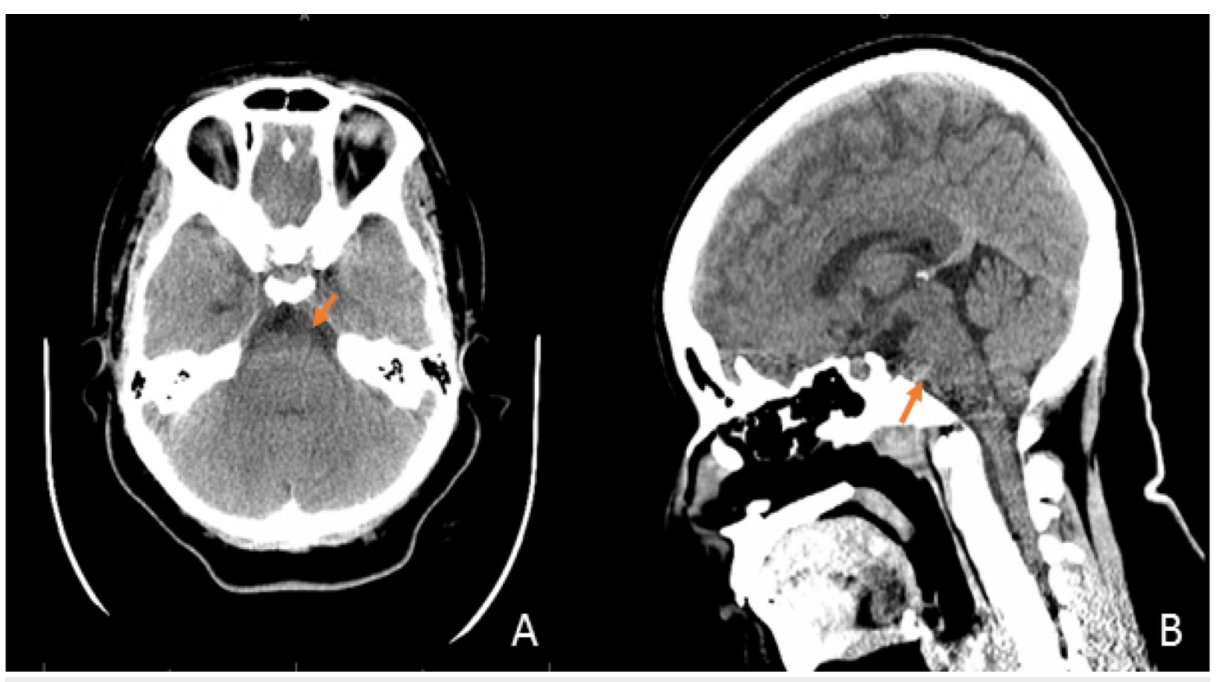

\section{FIGURE 1: CT head without contrast at admission}

CT head without contrast performed at admission: an isodense retroclival mass with mild mass effect on the pons (arrow). A: axial view; B: sagittal view. This was read as unremarkable before the brain MRI was performed

CT: computed tomography; MRI: magnetic resonance imaging

\begin{tabular}{|l|l|}
\hline CSF test & Result \\
\hline Cell count & WBC 1, RBC 0 \\
Chemistry & Glucose 52, protein 38 \\
Meningitis/encephalitis panel & Negative \\
Electrophoresis & Negative \\
Aquaporin-4 & Negative \\
Cytology & No malignant cells \\
\hline
\end{tabular}

\section{TABLE 1: CSF test results}

CSF: cerebrospinal fluid; WBC: white blood cells; RBC: red blood cells
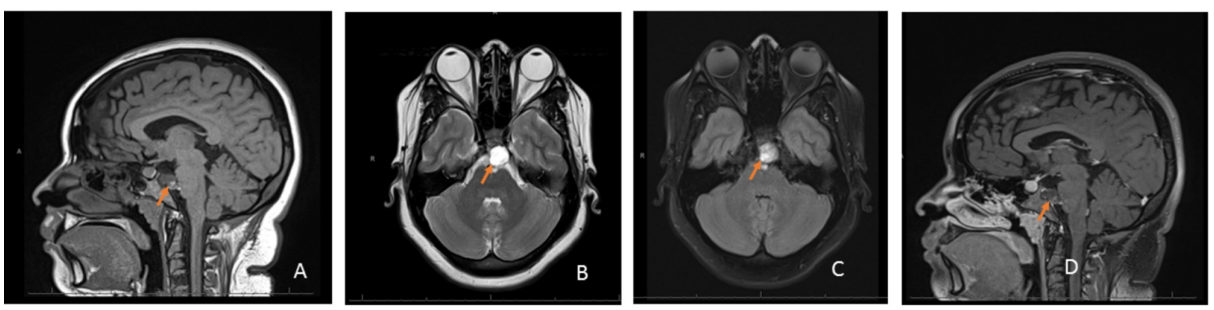


\section{Cureus}

\section{FIGURE 2: Brain MRI with and without contrast}

Brain MRI with and without contrast performed on admission. A - sagittal T1-weighted view: the mass appears heterogeneously hypointense (arrow); B - axial T2-weighted view: the mass appears homogenously hyperintense (arrow); C - axial FLAIR: the mass has slightly heterogeneously hyperintense diffusion (arrow); D - gadolinium-enhanced sagittal T1-weighted view: a lesion in the retroclival prepontine location with no contrast enhancement (arrow)

MRI: magnetic resonance imaging; FLAIR: fluid attenuation inversion recovery

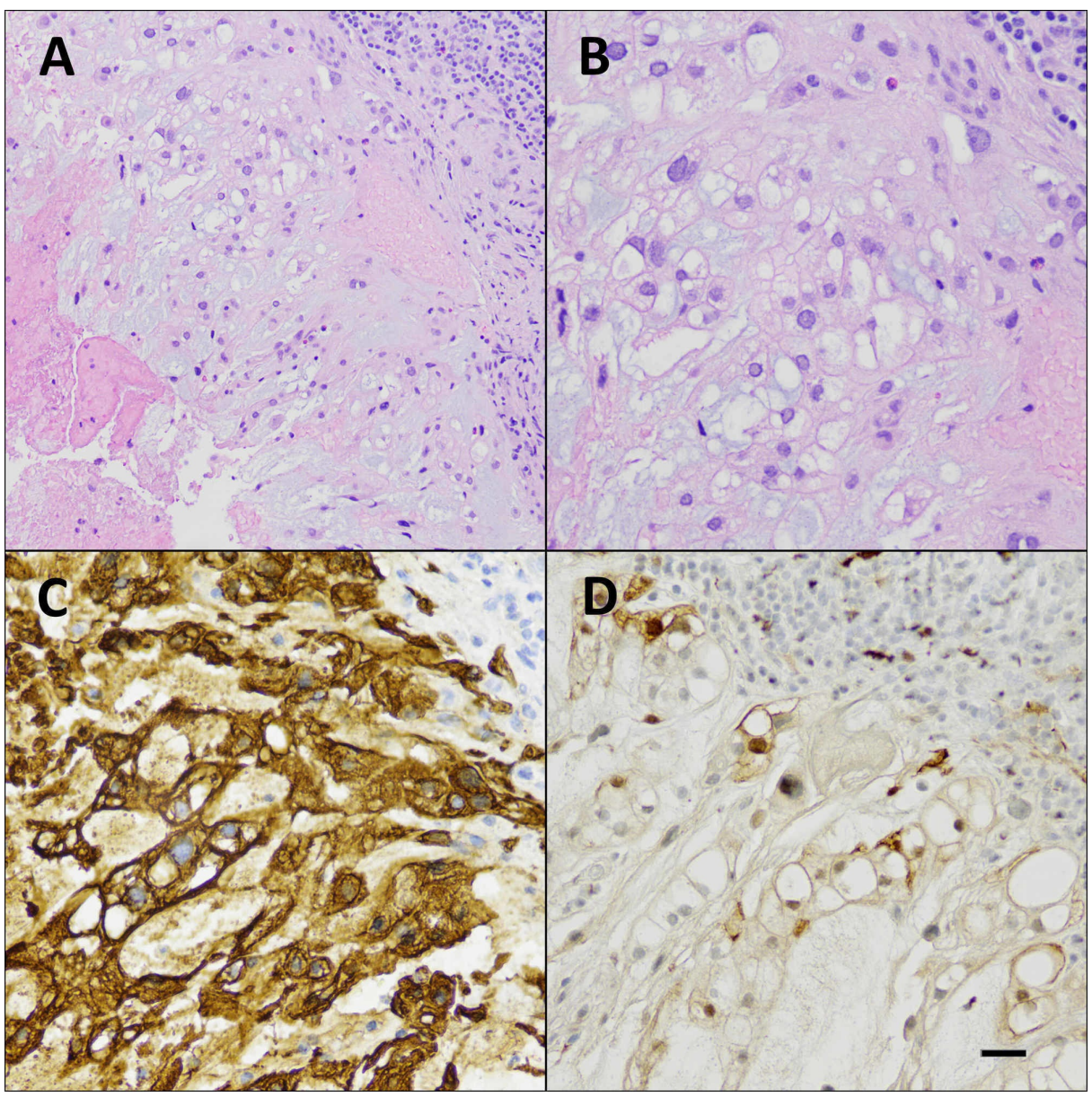

FIGURE 3: Histopathology of the retroclival mass

Low-magnification (A) and high-magnification (B) images of hematoxylin and eosin-stained, formalin-fixed, paraffin-embedded sections of the lesion, showing physaliphorous cells (center) and lymphocytic infiltrates in adjacent tissue included in the biopsy (upper right). Immunohistochemical stains for pancytokeratins (C) and $\mathrm{S} 100$ (D), showing strong reactivity of tumor cells for cytokeratins (AE1/AE3) and weak reactivity for S100. Scale bar (lower right) represents 50 microns for Figure 3A and 25 microns for Figures 3B-3D. Note that the distinction between chordoma and ecchordosis physaliphora is not readily determined solely from this histology but is dependent on biologic behavior including invasiveness

\section{Discussion}

Ecchordosis physaliphora is a congenital benign hamartomatous lesion. It is usually asymptomatic and incidentally found in $0.5-2 \%$ of autopsies. Our patient presented with an acute severe headache and suddenonset diplopia. Lumbar puncture demonstrated intracranial hypertension with an opening pressure of $37 \mathrm{~cm}$ $\mathrm{H}_{2} \mathrm{O}$. Given the brain MRI findings and follow-up outcomes, we believe that direct compression from the tumor and increased intracranial pressure contributed to the patient's symptoms.

The diagnosis of ecchordosis physaliphora can be challenging without the aid of an MRI. The initial head CT was read as normal in this case. In cases of intracranial hypertension and obesity, idiopathic intracranial hypertension is often atop the list of differential diagnoses. In this case, however, there was no papilledema on the fundoscopic exam. Brain MRI with and without contrast revealed the possible diagnosis of 
ecchordosis physaliphora with specific features: non-enhancing retroclival mass with increased signal intensity on T2-weighted sequences and decreased signal intensity on T1-weighted sequences $[1,8,9]$. The malignant counterpart, chordoma, usually presents as an enhancing retroclival mass with hyperintensity on both $\mathrm{T} 1$ and $\mathrm{T} 2$-weighted sequences, due to intra-tumoral calcification, increased vascularity, and disruption of the blood-brain barrier. Furthermore, the tissue analysis confirmed the diagnosis of ecchordosis physaliphora.

No previous case reports regarding ecchordosis physaliphora have mentioned raised opening pressure in the lumbar puncture. Our patient's initial brain MRI showed non-enhancing retroclival mass with mass effect upon the ventral pons and the left sixth cranial nerve stretch. The mass with a size of $17 \mathrm{~mm} \times 15 \mathrm{~mm} \times 19$ $\mathrm{mm}$ at the prepontine region might cause intracranial hypertension even though brain MRI did not show any radiographic features for increased intracranial pressure. While the patient did not have papilledema, we could not rule out idiopathic intracranial hypertension completely due to the patient's history, which was the reason why the patient was placed on Diamox initially.

A lesson learned from this case is that a brain MRI with and without contrast is essential for the diagnosis of ecchordosis physaliphora and that this diagnosis should be considered in cases that show similarity to idiopathic intracranial hypertension. In our patient, ecchordosis physaliphora could have been misdiagnosed as idiopathic intracranial hypertension had the MRI of the brain not been performed.

\section{Conclusions}

In this report, we discussed a rare case of symptomatic ecchordosis physaliphora presenting with headache, nausea, abducens nerve palsy, and intracranial hypertension. The diagnosis was revealed by brain MRI with and without contrast as well as tissue analysis.

\section{Additional Information \\ Disclosures}

Human subjects: Consent was obtained by all participants in this study. Conflicts of interest: In compliance with the ICMJE uniform disclosure form, all authors declare the following: Payment/services info: All authors have declared that no financial support was received from any organization for the submitted work. Financial relationships: All authors have declared that they have no financial relationships at present or within the previous three years with any organizations that might have an interest in the submitted work. Other relationships: All authors have declared that there are no other relationships or activities that could appear to have influenced the submitted work.

\section{References}

1. Lagman C, Varshneya K, Sarmiento JM, Turtz AR, Chitale RV: Proposed diagnostic criteria, classification schema, and review of literature of notochord-derived ecchordosis physaliphora. Cureus. 2016, 8:e547. 10.7759/cureus.547

2. Choudhri O, Feroze A, Hwang P, Vogel H, Ajlan A, Harsh G 4th: Endoscopic resection of a giant intradural retroclival ecchordosis physaliphora: surgical technique and literature review. World Neurosurg. 2014, 82:e21-26. 10.1016/j.wneu.2014.06.019

3. Ghimire P, Shapey J, Bodi I, Connor S, Thomas N, Barkas K: Spontaneous tension pneumocephalus and pneumoventricle in ecchordosis physaliphora: case report of a rare presentation and review of the literature (Epub ahead of print). Br J Neurosurg. 2019, 1-6. 10.1080/02688697.2019.1594695

4. Ilorah C, Bond B, Kattah JC, Hassanzadeh B: An incidental finding of ecchordosis physaliphora in a case of abducens nerve palsy: case report. Neuroophthalmology. 2017, 42:233-236. 10.1080/01658107.2017.1381853

5. Galloway L, Hayhurst C: Spontaneous cerebrospinal fluid rhinorrhoea with meningitis secondary to ecchordosis physaliphora. Br J Neurosurg. 2019, 33:99-100. 10.1080/02688697.2017.1297766

6. Ahn SS, Han J: Ecchordosis physaliphora presenting with abducens nerve palsy. J AAPOS. 2016, 20:266-268. 10.1016/j.jaapos.2016.01.010

7. Yamamoto T, Yano S, Hide T, Kuratsu J: A case of ecchordosis physaliphora presenting with an abducens nerve palsy: a rare symptomatic case managed with endoscopic endonasal transsphenoidal surgery. Surg Neurol Int. 2013, 4:13. 10.4103/2152-7806.106562

8. Park HH, Lee KS, Ahn SJ, Suh SH, Hong CK: Ecchordosis physaliphora: typical and atypical radiologic features. Neurosurg Rev. 2017, 40:87-94. 10.1007/s10143-016-0753-4

9. Mehnert F, Beschorner R, Küker W, Hahn U, Nägele T: Retroclival ecchordosis physaliphora: MR Imaging and review of the literature. AJNR Am J Neuroradiol. 2004, 25:1851-1855. 